SHORT REPORT

\title{
Somatic NKX2-5 mutations as a novel mechanism of disease in complex congenital heart disease
}

\author{
S M Reamon-Buettner, J Borlak
}

J Med Genet 2004;41:684-690. doi: 10.1136/jimg.2003.017483

NKX2-5 is a pivotal transcription factor in heart development. Previous studies on lymphocytic DNA provided evidence of familial NKX2-5 gene mutations in cardiac malformations. Common mutations are rare in unrelated families. We analysed, by direct sequencing, the gene encoding NKX2-5 in the diseased heart tissues of 68 patients with complex congenital heart disease, focussing particularly on atrial, ventricular, and atrioventricular septal defects. We identified 35 non-synonymous NKX2-5 mutations in the diseased heart tissues of patients. These mutations were mainly absent in normal, for example, unaffected, heart tissue of the same patient, indicating the somatic nature and mosaicism of mutations. We also observed multiple mutations and multiple haplotypes, as well as mutations in Down's syndrome patients with cardiac malformations. Taken collectively, the above results suggest the somatic nature of NKX2-5 mutations associated with complex cardiac malformations. Somatic mutations in transcription factor genes of cardiac progenitor cells provide a novel mechanism of disease.

$\mathrm{N}$ KX2-5 is a homeodomain containing transcription factor crucial to mammalian heart development. ${ }^{1}$ Lack of NKX2-5 in animal models results in lethality due to impaired cardiac looping. Genetic analysis of lymphocytic DNA identified germline mutations in the human NKX2-5 gene which are associated with cardiac anomalies, but most families have single and unique mutations. ${ }^{2-10}$ Germline NKX2-5 mutations are rare among sporadic cases of congenital heart disease (CHD), genotype-phenotype correlation is difficult, and mechanisms leading to complex CHD remain unclear.

Next to inherited mutations, the importance of somatic mutations during early embryogenesis is now being recognised, but rarely studied. ${ }^{11}{ }^{12}$ Disease associated or disease causing somatic mutations are undetected by genetic analysis of lymphocytic DNA alone, and mosaicism may reduce the likelihood of detection in the affected tissue. Therefore, mutations present in cardiac tissues may be absent or sporadic in blood samples of the same individual. Unlike previous studies, we searched for mutations in the diseased tissues of 68 explanted hearts of patients with definitive atrial (ASD), ventricular (VSD), and atrioventricular (AVSD) septal defects. These patients, however, had complex cardiac malformations and died mostly in early infancy (fig lA and B).

The human NKX2-5 gene maps to chromosome 5 q34 and consists of two exons encoding a protein of 324 amino acids. The homeodomain (HD) of NKX2-5, which lies within exon 2 , consists of three $\alpha$ helices. Helix 3 is important for DNA binding specificity. ${ }^{13}$ Other conserved regions of NKX2-5 are the TN domain and NK2 specific domain (NK2-SD). Using validated PCR primers, ${ }^{3}{ }^{5}$ we amplified fragments from the two exons (fig 1C). Mutations were detected by direct sequencing of both DNA strands and confirmed by PCRRFLP or cloning of the sequenced fragments, allowing separation of the two variant alleles in a heterozygous locus (fig 1D).

We identified two NCBI dbSNPs (rs2277923 and rs703752) and 35 non-synonymous NKX2-5 mutations in diseased heart tissues of patients with complex cardiac malformations (table 1). Three of these mutations (Arg25Cys, Thr178Met, and Ala219Val) are known. We detected 29 mutations in VSD patients, 12 in ASD, and 14 in AVSD. We found one mutation in the TN domain (Asn19Ser) and five mutations (Lys183Glu, Gln187Ter, Lys 192Thr, Lys192Arg, Lys194Arg) in the third helix of HD (table 1). We observed only heterozygous genotypes based on the sequence electropherograms. As determined by haplotype analysis, this heterozygosity may simply be a reflection of genotypes of mixed wild type and mutant cells. Therefore, the occurrence of homozygous genotypes in analysed diseased heart tissues cannot be excluded, although germline homozygous mutations in animal models are lethal. Further, somatically occurring mutations as seen in cancerous diseases result in loss of heterozygosity. Again, such loss of heterozygosity may result in cardiac anomalies as seen in our study. The 35 mutations were absent in lymphocytic DNA of 50 healthy individuals and in DNA from six frozen normal hearts.

The explanted hearts were formalin fixed more than 40 years ago, but analysis of NKX2-5 and five other cardiac transcription factor genes (46 $709 \mathrm{nt}$ ) in 10 normal hearts from the same collection of formalin fixed hearts basically revealed no sequence alterations except dbSNPs, showing the reliability of using valuable archival material. We found a non-synonymous NKX2-5 substitution (T984A, Cys270Ser) in two individuals who died as babies, but this mutation was different from those in patients with CHD. This sequence alteration may just be a polymorphism.

The NKX2-5 mutations were detected in heart tissues sampled within the septal defects of the hearts. To determine whether these mutations were also present in normal heart tissues (such as in patients with VSD, where tissue samples were taken from unaffected atria), we compared 27 distinct mutations in normal and diseased heart tissues of the same patients (table 1). We analysed a total of 92 samples, 146 PCR fragments, and 77598 nucleotides and found mutations mainly absent in normal heart tissues. The absence of mutations in matched normal heart tissue of the same patient indicates the somatic nature and mosaicism of NKX2-5 mutations.

AVSD is highly associated with Down's syndrome (trisomy $21)^{14}$ and some of our patient cohorts had Down's syndrome.

Abbreviations: ASD, atrial septal defect; AVSD, atrioventricular septal defect; CHD, congenital heart disease; HD, homeodomain; VSD, ventricular septal defect 
A

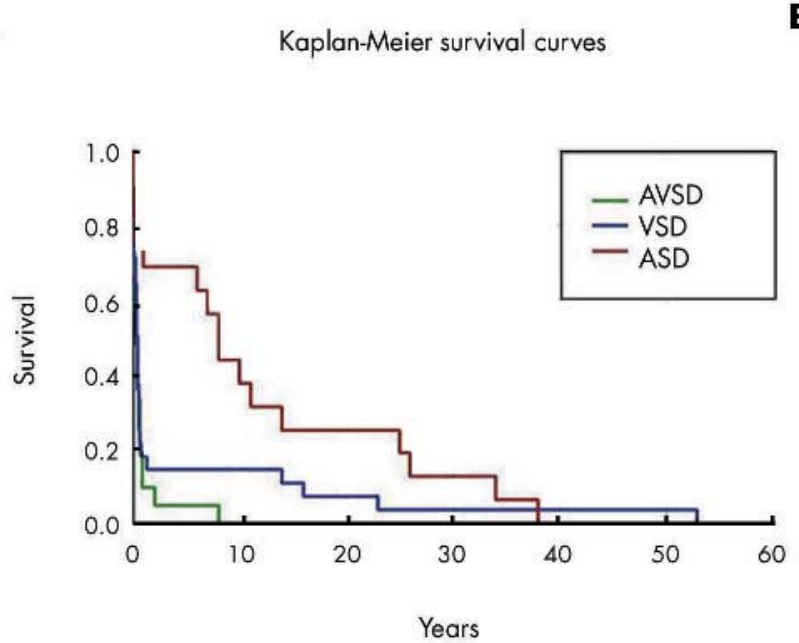

C

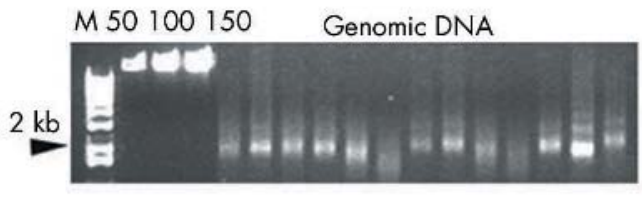

1F24/1AR

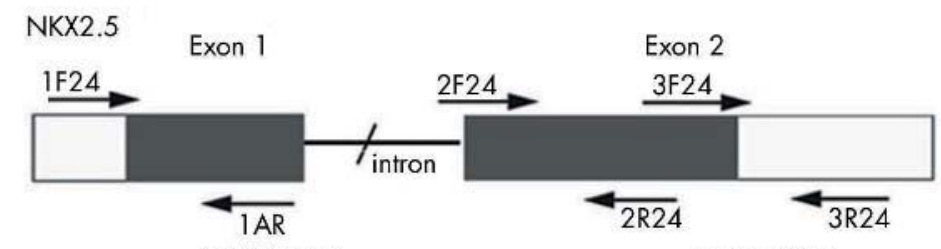

$2 F 24 / 2 R 24$

Subaortic (2a) and Septum secundum (1) and muscular (2b) VSD septum primum (4) ASD
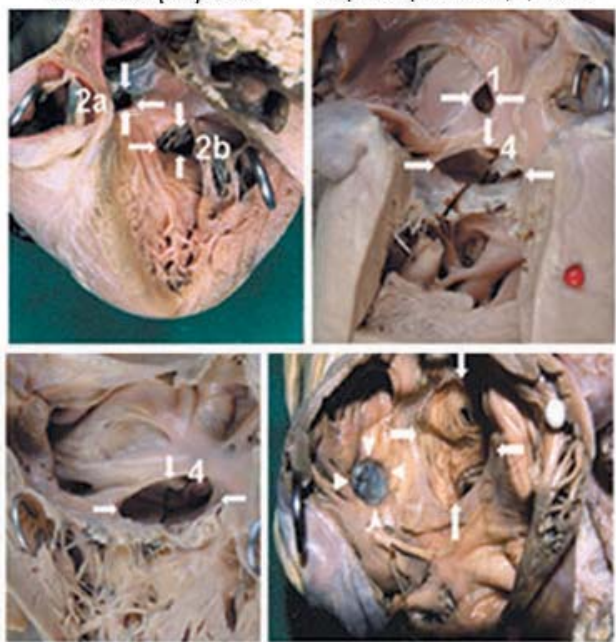

Septum primum ASD Septum primum ASD (arrows) and tricuspid atresia (arrow heads)

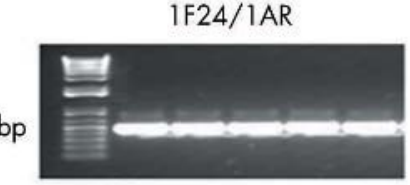

$485 \mathrm{bp}$

D
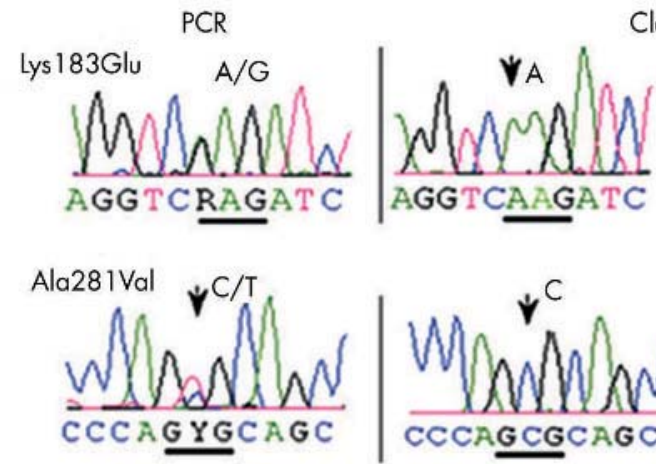

Clones

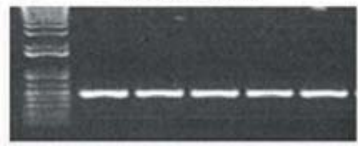

$3 F 24 / 3 R 24$

$573 \mathrm{bp}$

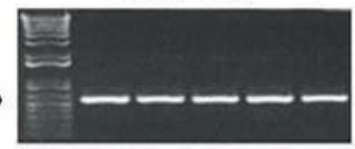

Figure 1 NKX2-5 mutation analysis in complex CHD. (A) Kaplan-Meier survival curves showing AVSD and VSD patients with lower life expectancy than ASD patients. (B) Examples of cardiac malformations in patients. (C) Three NKX2-5 fragments were amplified from genomic DNA (size of about 2 $\mathrm{kb})$ isolated from archival cardiac tissues using three known primer pairs: 1F24/1AR (485 bp), 2F/2R24 (472 bp), and 3F/3R24 (573 bp). About 20-50 ng genomic DNA was used for PCR reaction. M (kb ladder): 50, 100, $150 \mathrm{ng}$ ( $\lambda$ DNA standard). (D) Detection of mutations by direct sequencing, verification by PCR-RFLP, or cloning of fragments with heterozygous loci, separating the two variant alleles.

Analysis of 18 AVSD patients with Down's syndrome for NKX2-5 mutations found no difference in the mutation spectrum between syndromic and non-syndromic AVSD patients (tables 1 and 2). The occurrence of both trisomy 21 and NKX2-5 mutations suggests genomic chaos in the heart tissues of patients with complex CHD. We also detected common mutations among unrelated patients and some mutations were specific to ventricular or atrioventricular septal defects (table 2). We found common mutations such as A232G (Asn19Ser), A723G (Lys183Glu), C1012T (Ser279Phe), C1018T (Ala281Val), and Al072G (Asp299Gly) (table 2). We also found multiple mutations in 57 patients; $66 \%$ had five or more mutations. The range of multiple mutations per patient was 2-14, 2-7, and 2-10 in VSD, ASD, and AVSD patients, respectively (table 2).

The presence of multiple gene mutations in a patient is an unlikely occurrence in inherited mutations, but multiple mutations are frequently observed in high capacity cell 
Table 1 Summary of NKX2-5 mutations

\begin{tabular}{|c|c|c|c|c|c|c|c|}
\hline $\begin{array}{l}\text { Nucleotide change } \\
\text { (NKX2-5 } \\
\text { NM004387) }\end{array}$ & $\begin{array}{l}\text { Nucleotide } \\
\text { reference } \\
\text { (coding region) }\end{array}$ & $\begin{array}{l}\text { Amino acid } \\
\text { change }\end{array}$ & Location & $\begin{array}{l}\text { Number of } \\
\text { positive hearts } \\
(n=68)\end{array}$ & $\begin{array}{l}\text { Mutations compared } \\
\text { in normal } v \text { diseased } \\
\text { heart tissues }\end{array}$ & $\begin{array}{l}\text { Mutations detected in } \\
\text { both Down's and } \\
\text { non-Down's syndrome } \\
\text { AVSD patients }\end{array}$ & $\begin{array}{l}\text { PCR-RFLP } \\
\text { assay }\end{array}$ \\
\hline \multicolumn{8}{|l|}{ Non-synonymous } \\
\hline $196 \mathrm{~T} \rightarrow \mathrm{C}$ & 20 & 7 Leu $\rightarrow$ Pro & Exon 1 & 1 & & & \\
\hline $232 \mathrm{~A} \rightarrow \mathrm{G}$ & 56 & $19 \mathrm{Asn} \rightarrow \mathrm{Ser}$ & Exon $1, \mathrm{TN}$ domain & 18 & & & \\
\hline $249 \mathrm{C} \rightarrow \mathrm{T}$ & 73 & $25 \mathrm{Arg} \rightarrow$ Cys & Exon 1 & 1 & & & Hhal \\
\hline $309 \mathrm{~T} \rightarrow \mathrm{C}$ & 133 & $45 \mathrm{Ser} \rightarrow$ Pro & Exon 1 & 1 & & & \\
\hline $327 \mathrm{~T} \rightarrow \mathrm{C}$ & 151 & $51 \mathrm{Phe} \rightarrow$ Leu & Exon 1 & 1 & & & \\
\hline $382 \mathrm{~T} \rightarrow \mathrm{C}$ & 206 & $69 \mathrm{Leu} \rightarrow$ Pro & Exon 1 & 1 & & & \\
\hline $406 \mathrm{C} \rightarrow \mathrm{T}$ & 230 & 77 Pro $\rightarrow$ Leu & Exon 1 & 1 & & & \\
\hline $516 \mathrm{~T} \rightarrow \mathrm{A}$ & 340 & $114 \mathrm{Cys} \rightarrow$ Ser & Exon 2 & 5 & & $\times$ & \\
\hline $516 \mathrm{~T} \rightarrow \mathrm{C}$ & 340 & 114 Cys $\rightarrow$ Arg & Exon 2 & 22 & $\times$ & $\times$ & \\
\hline $529 \mathrm{~A} \rightarrow \mathrm{G}$ & 353 & 118 Lys $\rightarrow$ Arg & Exon 2 & 5 & $x$ & & \\
\hline $547 \mathrm{~A} \rightarrow \mathrm{G}$ & 371 & 124 Lys $\rightarrow$ Arg & Exon 2 & 6 & $\hat{x}$ & & \\
\hline $553 \mathrm{~A} \rightarrow \mathrm{T}$ & 377 & $126 \mathrm{Glu} \rightarrow \mathrm{Val}$ & Exon 2 & 11 & $\hat{x}$ & $\times$ & \\
\hline $573 \mathrm{C} \rightarrow \mathrm{T}$ & 397 & 133 Pro $\rightarrow$ Ser & Exon 2 & 6 & $\hat{x}$ & & \\
\hline $579 \mathrm{G} \rightarrow \mathrm{A}$ & 403 & $135 \mathrm{Ala} \rightarrow \mathrm{Thr}$ & Exon 2 & 9 & $\times$ & $x$ & Aval \\
\hline $607 \mathrm{~T} \rightarrow \mathrm{C}$ & 431 & 144 Leu $\rightarrow$ Pro & Exon 2 & 11 & $\times$ & $x$ & \\
\hline $709 \mathrm{C} \rightarrow \mathrm{T}$ & 533 & $178 \mathrm{Thr} \rightarrow$ Met & Exon 2, HD & 5 & $\times$ & & Nlalll \\
\hline $723 \mathrm{~A} \rightarrow \mathrm{G}$ & 547 & 183 Lys $\rightarrow$ Glu & $\begin{array}{l}\text { Exon 2, HD, third } \\
\text { helix }\end{array}$ & 29 & $\times$ & $x$ & Taql \\
\hline $735 \mathrm{C} \rightarrow \mathrm{T}$ & 559 & $187 \mathrm{Gln} \rightarrow \mathrm{Ter}$ & $\begin{array}{l}\text { Exon 2, HD, third } \\
\text { helix }\end{array}$ & 6 & $x$ & & Xbal \\
\hline $751 \mathrm{~A} \rightarrow \mathrm{C}$ & 575 & 192 Lys $\rightarrow$ Thr & $\begin{array}{l}\text { Exon 2, HD, third } \\
\text { helix }\end{array}$ & 6 & $\times$ & & Tail \\
\hline $751 \mathrm{~A} \rightarrow \mathrm{G}$ & 575 & 192 Lys $\rightarrow$ Arg & $\begin{array}{l}\text { Exon 2, HD, third } \\
\text { helix }\end{array}$ & 2 & $\times$ & & $B f m l$ \\
\hline $757 \mathrm{~A} \rightarrow \mathrm{G}$ & 581 & 194 Lys $\rightarrow$ Arg & $\begin{array}{l}\text { Exon 2, HD, third } \\
\text { helix }\end{array}$ & 2 & $x$ & & Bsgl \\
\hline $790 \mathrm{~T} \rightarrow \mathrm{A}$ & 614 & $205 \mathrm{Val} \rightarrow$ Glu & Exon 2 & 6 & $\times$ & & \\
\hline $832 \mathrm{C} \rightarrow \mathrm{T}$ & 656 & $219 \mathrm{Ala} \rightarrow \mathrm{Val}$ & Exon 2, NK2-SD & 10 & $\times$ & & \\
\hline $852 \mathrm{G} \rightarrow \mathrm{A}$ & 676 & 226 Asp $\rightarrow$ Asn & Exon 2 & 2 & $x$ & & \\
\hline $918 \mathrm{~T} \rightarrow \mathrm{C}$ & 742 & $248 \mathrm{Tyr} \rightarrow \mathrm{His}$ & Exon 2 & 5 & $\times$ & & \\
\hline $1011 \mathrm{~T} \rightarrow \mathrm{C}$ & 835 & $279 \mathrm{Ser} \rightarrow$ Pro & Exon 2 & 3 & $\times$ & & \\
\hline $1012 \mathrm{C} \rightarrow \mathrm{T}$ & 836 & $279 \mathrm{Ser} \rightarrow \mathrm{Phe}$ & Exon 2 & 23 & $\times$ & $x$ & \\
\hline $1018 \mathrm{C} \rightarrow \mathrm{T}$ & 842 & $281 \mathrm{Ala} \rightarrow \mathrm{Val}$ & Exon 2 & 34 & $\hat{x}$ & $\hat{x}$ & $B m r l$ \\
\hline $1033 \mathrm{C} \rightarrow \mathrm{T}$ & 857 & $286 \mathrm{Ala} \rightarrow \mathrm{Val}$ & Exon 2 & 22 & $\times$ & $x$ & \\
\hline $1056 \mathrm{~A} \rightarrow \mathrm{C}$ & 880 & $294 \mathrm{Asn} \rightarrow \mathrm{His}$ & Exon 2 & 14 & $\times$ & $\times$ & \\
\hline $1072 \mathrm{~A} \rightarrow \mathrm{G}$ & 896 & 299 Asp $\rightarrow$ Gly & Exon 2 & 36 & $\times$ & $x$ & \\
\hline $1089 \mathrm{~A} \rightarrow \mathrm{G}$ & 913 & 305 Ser $\rightarrow$ Gly & Exon 2 & 1 & $\times$ & & \\
\hline $1134 \mathrm{G} \rightarrow \mathrm{A}$ & 958 & 320 Gly $\rightarrow$ Ser & Exon 2 & 17 & $\hat{x}$ & & \\
\hline $1141 \mathrm{G} \rightarrow \mathrm{A}$ & 965 & $322 \mathrm{Arg} \rightarrow \mathrm{Gln}$ & Exon 2 & 2 & $x$ & & \\
\hline $\begin{array}{l}1149 \mathrm{~T} \rightarrow \mathrm{C} \\
\mathrm{dbSNPs}\end{array}$ & 973 & $\mathrm{Stop} \rightarrow \mathrm{Gln}$ & Exon 2 & 12 & $x$ & $x$ & \\
\hline $\begin{array}{l}239 \mathrm{~A} \rightarrow \mathrm{G} \\
1212 \mathrm{~T} \rightarrow \mathrm{G}\end{array}$ & 63 & $21 \mathrm{Glu}$ & $\begin{array}{l}\text { Exon 1, rs2277923 } \\
\text { Exon 2, rs703752 }\end{array}$ & $\begin{array}{l}\text { 8AA: } 55 \text { AG } \\
13 \text { GG: } 53 \text { GT }\end{array}$ & & & Bpml \\
\hline
\end{tabular}

division such as in cancer. ${ }^{15}$ For example, mutational analysis of the p53 tumour suppressor gene in human mammary carcinomas evidenced up to six mutations in this gene. ${ }^{16}$ It is, however, uncommon to find carriers of multiple mutations within one gene, as described here. We observed, on average, five mutations in VSD and AVSD subjects, and three mutations in ASD subjects. Moreover, nine of 29 VSD subjects, nine of 23 AVSD subjects, and three of 16 ASD subjects had more than five mutations. Nonetheless, recent genetic studies also demonstrate the occurrence of multiple mutations in human genes including the genes coding for butyrylcholinesterase $\mathrm{P}^{17}$ and hALG6, which codes for a glucosyltransferase. ${ }^{18}$ In both genes, three mutations per patient were identified. In our study, it remains unclear whether multiple NKX2-5 mutations are a result of primary or secondary events.

We cloned PCR products containing heterozygous loci, as seen on sequence electropherograms. In most cases, a locus was scored heterozygous when the ratio of the second peak to the highest peak was at least 50\%. After cloning, we sequenced random clones, and scored only those loci previously identified as heterozygous after direct sequencing of the PCR product. Surprisingly, we obtained more than the two expected haplotypes in one individual, and identified clones carrying all the reference alleles only, mutated alleles only, or a combination of both (table 3 ). In most analysed clones, both alleles were affected. A likely explanation for multiple haplotypes would be a mixed population of cardiomyocytes carrying different mutations or de novo chromosomal rearrangements and gene duplications in the heart tissues of patients affected by various mutations. Heart defects are observed following chromosomal abnormalities including chromosome $5 .{ }^{19}$ It will be the subject of future research to determine the origin of these multiple haplotypes.

Our patient cohort of rare and complex cardiac malformations had non-synonymous mutations in other cardiac transcription factor genes including GATA4, HANDl, and TBX5, although mutation frequencies varied considerably (data not shown). Simultaneous occurrence of multiple mutations in several genes is uncommon, but may be explained by the sheer complexity of the observed malformations, with patients carrying multiple defects in the heart. Somatically occurring mutations may affect various cell types at different stages of organogenesis, with different transcription factor programs taking place. These patients, however, did not carry mutations in the sequences affecting binding regions of $\mathrm{MEF} 2 \mathrm{C}$, an upstream determinant of cardiac development. ${ }^{20}$ NKX2-5, HAND1, and MEF2C are all located 


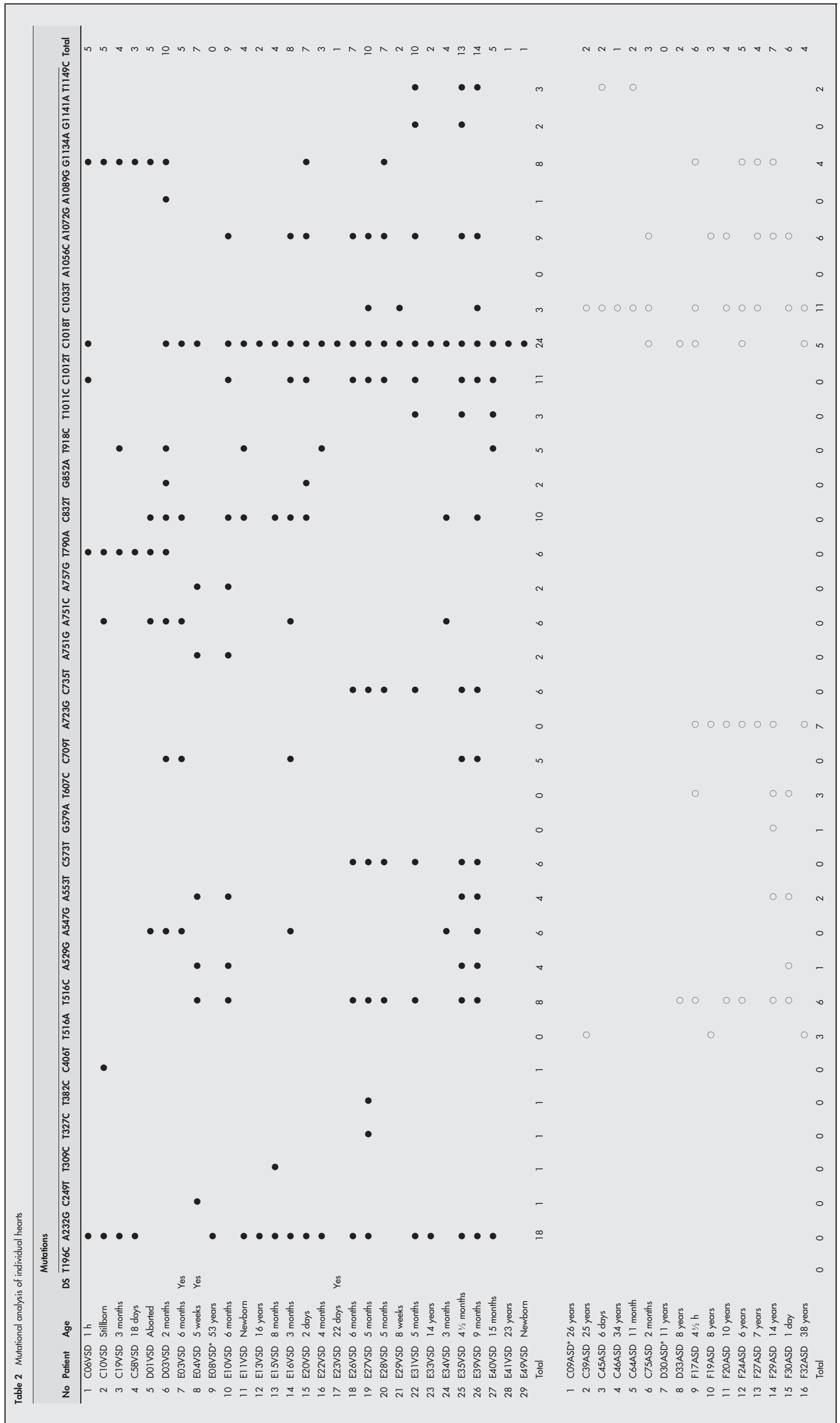




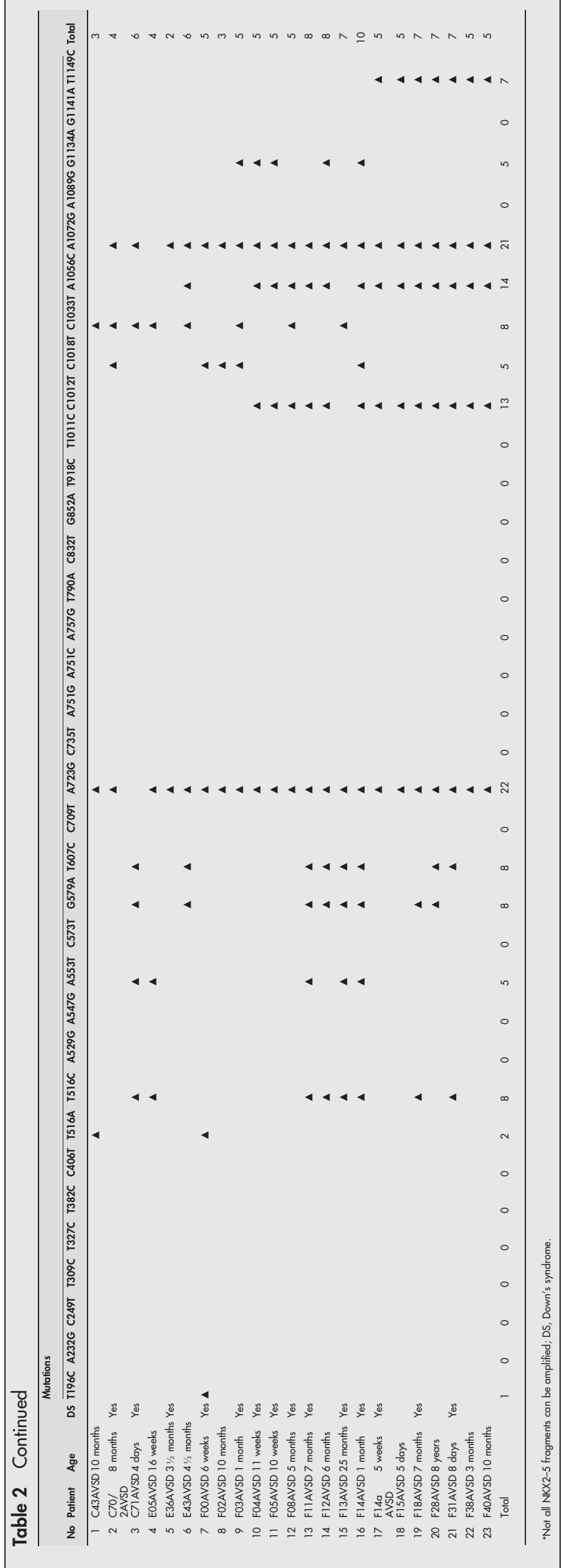


Table 3 Multiple NKX2-5 haplotypes in complex cardiac malformations

\begin{tabular}{|c|c|c|c|c|c|c|c|c|c|c|c|c|}
\hline \multirow[b]{2}{*}{ Patients } & \multicolumn{11}{|c|}{ Mutations } & \multirow[b]{2}{*}{ Total } \\
\hline & T516C & A529G & A547G & A553T & C573T & C709T & C735T & A751C & A751G & A757G & C832T & \\
\hline E04VSD & & & & & & & & & & & & 2 \\
\hline PCR & & A/G & & $A / T$ & & & & & $A / G$ & A/G & & \\
\hline Clone 1 & & A & & $A$ & & & & & A & A & & $\begin{array}{l}\text { Haplotype 1, all } \\
\text { reference alleles }\end{array}$ \\
\hline Clone 2 & & G & & T & & & & & G & G & & $\begin{array}{l}\text { Haplotype 2, all } \\
\text { mutant alleles }\end{array}$ \\
\hline Clone 3 & & G & & $\mathrm{T}$ & & & & & G & G & & \\
\hline Clone 4 & & G & & $\mathrm{T}$ & & & & & G & G & & \\
\hline Clone 5 & & G & & $\mathrm{T}$ & & & & & G & G & & \\
\hline Clone 6 & & $G$ & & $\mathrm{~T}$ & & & & & $G$ & $G$ & & \\
\hline E10VSD & & & & & & & & & & & & 3 \\
\hline PCR & & $A / G$ & & $A / T$ & & & & & $A / G$ & A/G & & \\
\hline Clone 1 & & A & & A & & & & & A & A & & $\begin{array}{l}\text { Haplotype 1, all } \\
\text { reference alleles }\end{array}$ \\
\hline Clone 2 & & A & & A & & & & & A & A & & \\
\hline Clone 3 & & A & & $\mathrm{T}$ & & & & & $G$ & G & & Haplotype 2, \\
\hline Clone 4 & & $G$ & & $\mathrm{~T}$ & & & & & G & G & & $\begin{array}{l}\text { Haplotype 3, } \\
\text { all mutant alleles }\end{array}$ \\
\hline Clone 5 & & G & & $\mathrm{T}$ & & & & & G & G & & \\
\hline Clone 6 & & G & & $\mathrm{T}$ & & & & & G & G & & \\
\hline EO3VSD & & & & & & & & & & & & 3 \\
\hline PCR & & & $A / G$ & & & $\mathrm{C} / \mathrm{T}$ & & $\mathrm{A} / \mathrm{C}$ & & & $\mathrm{C} / \mathrm{T}$ & \\
\hline Clone 1 & & & A & & & C & & A & & & C & $\begin{array}{l}\text { Haplotype 1, all } \\
\text { reference alleles }\end{array}$ \\
\hline Clone 2 & & & A & & & c & & A & & & C & \\
\hline Clone 3 & & & G & & & c & & C & & & $\mathrm{T}$ & Haplotype 2 \\
\hline Clone 4 & & & G & & & $\mathrm{T}$ & & C & & & $T$ & $\begin{array}{l}\text { Haplotype } 3 \text {, } \\
\text { all mutant alleles }\end{array}$ \\
\hline D03VSD & & & & & & & & & & & & 3 \\
\hline & & & $A / G$ & & & $\mathrm{C} / \mathrm{T}$ & & $\mathrm{A} / \mathrm{C}$ & & & $\mathrm{C} / \mathrm{T}$ & \\
\hline Clone 1 & & & G & & & C & & A & & & C & $\begin{array}{l}\text { Haplotype 1, all } \\
\text { reference alleles }\end{array}$ \\
\hline Clone 2 & & & A & & & C & & A & & & $\mathrm{T}$ & Haplotype 2 \\
\hline Clone 3 & & & G & & & $\mathrm{T}$ & & C & & & $T$ & $\begin{array}{l}\text { Haplotype 3, } \\
\text { all mutant alleles }\end{array}$ \\
\hline E3IVSD & & & & & & & & & & & & 4 \\
\hline PCR & $\mathrm{T} / \mathrm{C}$ & & & & $\mathrm{C} / \mathrm{T}$ & & $\mathrm{C} / \mathrm{T}$ & & & & & \\
\hline Clone 1 & $\mathbf{T}$ & & & & C & & C & & & & & $\begin{array}{l}\text { Haplotype 1, all } \\
\text { reference alleles }\end{array}$ \\
\hline Clone 2 & $\mathbf{T}$ & & & & C & & C & & & & & \\
\hline Clone 3 & $\mathrm{~T}$ & & & & c & & c & & & & & \\
\hline Clone 4 & $\mathrm{~T}$ & & & & c & & $\mathrm{T}$ & & & & & Haplotype 2 \\
\hline Clone 5 & $\mathrm{~T}$ & & & & c & & $\mathrm{T}$ & & & & & \\
\hline Clone 6 & $\mathrm{~T}$ & & & & c & & $\mathrm{T}$ & & & & & \\
\hline Clone 7 & $\mathrm{~T}$ & & & & c & & $\mathrm{T}$ & & & & & \\
\hline Clone 8 & $\mathrm{~T}$ & & & & $\mathrm{~T}$ & & C & & & & & Haplotype 3 \\
\hline Clone 9 & C & & & & $\mathrm{T}$ & & c & & & & & Haplotype 4 \\
\hline Clone 10 & C & & & & $\mathrm{T}$ & & C & & & & & \\
\hline E35VSD & & & & & & & & & & & & 5 \\
\hline $\begin{array}{l}\text { PCR } \\
\text { Clone } 1\end{array}$ & & & & & $\begin{array}{l}\mathrm{C} / \mathrm{t} \\
\mathrm{C}\end{array}$ & $\begin{array}{l}\mathrm{C} / \mathrm{t} \\
\mathrm{C}\end{array}$ & $\begin{array}{l}\mathrm{C} / \mathrm{t} \\
\mathrm{C}\end{array}$ & & $\begin{array}{l}A / g \\
A\end{array}$ & $\begin{array}{l}A / g \\
A\end{array}$ & & $\begin{array}{l}\text { Haplotype 1, all } \\
\text { reference alleles }\end{array}$ \\
\hline Clone 2 & & & & & C & C & C & & A & A & & \\
\hline Clone 3 & & & & & C & C & C & & A & A & & \\
\hline Clone 4 & & & & & C & C & $\mathrm{T}$ & & A & A & & Haplotype 2 \\
\hline Clone 5 & & & & & C & C & C & & G & G & & Haplotype 3 \\
\hline Clone 6 & & & & & $\mathrm{~T}$ & C & $\mathrm{T}$ & & A & A & & Haplotype 4 \\
\hline Clone 7 & & & & & T & C & T & & A & A & & \\
\hline $\begin{array}{l}\text { Clone } 8 \\
\text { E39VSD }\end{array}$ & & & & & T & T & C & & A & A & & $\begin{array}{l}\text { Haplotype } 5 \\
7\end{array}$ \\
\hline PCR & $\mathrm{T} / \mathrm{C}$ & $A / g$ & $A / g$ & $A / t$ & $C / t$ & $C / t$ & $C / t$ & $A / c / g$ & $A / c / g$ & $A / g$ & $C / t$ & \\
\hline Clone 1 & C & & A & A & $\mathrm{C}$ & $\mathrm{C}$ & C & & & A & c & Haplotype 1 \\
\hline Clone 2 & $T$ & A & G & A & C & C & C & A & & A & C & Haplotype 2 \\
\hline Clone 3 & C & G & A & $\mathrm{T}$ & C & $\mathrm{T}$ & C & A & & A & $T$ & Haplotype 3 \\
\hline Clone 6 & $\mathbf{T}$ & A & A & A & C & C & C & C & & A & $\mathrm{T}$ & Haplotype 4 \\
\hline Clone 5 & $\mathbf{T}$ & A & A & $\mathrm{T}$ & C & T & C & C & & A & C & Haplotype 5 \\
\hline Clone 4 & $\mathbf{T}$ & A & G & A & C & $\mathrm{T}$ & C & C & & A & T & Haplotype 6 \\
\hline Clone 7 & $\mathbf{T}$ & A & A & A & C & C & C & & G & G & C & Haplotype 7 \\
\hline Clone 8 & $T$ & A & A & A & C & C & C & & G & G & C & \\
\hline
\end{tabular}

on chromosome 5: 5q34,5q32, and 5q14, respectively, but differ profoundly in the spectrum of mutation. Deleterious mutations in MEF2C would most likely lead to early termination of pregnancy, for example, intrauterine resorption or spontaneous abortion due to its pivotal function in the transdifferentiation process during organogenesis of the heart.

We also analysed lymphocytic DNA in seven families and four unrelated patients with CHD, but did not identify 
NKX2-5 mutations, except for one synonymous substitution in an unaffected family member. Further sequencing of nine cardiac transcription factor genes evidenced only dbSNPs. Thus, analysis of lymphocytic DNA may not reveal the genetic aberrations which are associated with CHD. In conclusion, our results demonstrate absence of mutations in matched normal heart tissues of the same patients' hearts, multiple mutations as well as multiple haplotypes in patients. These observations strongly suggest that NKX2-5 mutations occur somatically and that such somatic mutations are associated with complex CHD. These mutations probably resulted from postzygotic errors in cell division, particularly in cardiac progenitors cells. Presumably, various cell types at different stages of organogenesis with different transcription factor programs in place are affected by somatic mutations. Determining the onset of somatic mutations, effects of multiple mutations, and breakdown of transcription factor crosstalks resulting from genomic instability in cardiac cells, will be an interesting avenue to explore in future stem cell research.

\section{ACKNOWLEDGEMENTS}

We thank the Institute of Anatomy, University of Leipzig for providing the heart collection; the Department of Cardiac Surgery and Pediatric Cardiology, University of Mainz for the blood samples of patients with CHD; and Yvonne Becker and Andreas Hiemisch for the excellent technical support.

\section{Authors' affiliations \\ S M Reamon-Buettner, J Borlak, Drug Research and Medical Biotechnology, Fraunhofer Institute of Toxicology and Experimental Medicine, Nikolai-Fuchs-Strasse 1, D-30625 Hanover, Germany \\ Financial support to JB from the Lower Saxony Ministry of Science and Culture is greatly appreciated. \\ Conflict of interest: none declared. \\ Correspondence to: J Borlak, Drug Research and Medical Biotechnology, Fraunhofer Institute of Toxicology and Experimental Medicine, Nikolai-Fuchs-Strasse 1, D-30625 Hanover, Germany; borlak@item.fraunhofer.de}

Revised version received 9 April 2004

Accepted for publication 13 April 2004

\section{REFERENCES}

1 Harvey RP, Lai D, Elliott D, Biben C, Solloway M, Prall O, Stennard F Schindeler A, Groves N, Lavulo L, Hyun C, Yeoh T, Costa M, Furtado M, Kirk E. Homeodomain factor Nkx2-5 in heart development and disease. Cold Spring Harb Symp Quant Biol 2002;67:107-14.
2 Schott JJ, Benson DW, Basson CT, Pease W, Silberbach GM, Moak JP Maron BJ, Seidman CE, Seidman JG. Congenital heart disease caused by mutations in the transcription factor NKX2-5. Science 1998;281(5373):108-11.

3 Benson DW, Silberbach GM, Kavanaugh-McHugh A, Cottrill C, Zhang Y, Riggs S, Smalls O, Johnson MC, Watson MS, Seidman JG, Seidman CE, Plowden J, Kugler JD. Mutations in the cardiac transcription factor NKX2-5 affect diverse cardiac developmental pathways. J Clin Invest 1999:104(1 1):1567-73.

4 Hosoda T, Komuro I, Shiojima I, Hiroi Y, Harada M, Murakawa Y, Hirata Y, Yazaki $Y$. Familial atrial septal defect and atrioventricular conduction disturbance associated with a point mutation in the cardiac homeobox gene CSX/NKX2-5 in a Japanese patient. Jpn Circ J 1999;63(5):425-6.

5 Goldmuntz E, Geiger E, Benson DW. NKX2-5 mutations in patients with tetralogy of fallot. Circulation 2001;104(21):2565-8.

6 Ikeda Y, Hiroi Y, Hosoda T, Utsunomiya T, Matsuo S, Ito T, Inove J, Sumiyoshi T, Takano H, Nagai R, Komuro I. Novel point mutation in the cardiac transcription factor CSX/NKX2-5 associated with congenital heart disease. Circ J 2002;66(6):561-3.

7 Watanabe Y, Benson DW, Yano S, Akagi T, Yoshino M, Murray JC. Two novel frameshift mutations in NKX2-5 result in novel features including visceral inversus and sinus venosus type ASD. J Med Genet 2002;39(1 1):807-1 1

8 Gutierrez-Roelens I, Sluysmans T, Gewillig M, Devriendt K, Vikkula M. Progressive AV-block and anomalous venous return among cardiac anomalies associated with two novel missense mutations in the CSX/NKX2-5 gene. Hum Mutat 2002;20:75-6.

9 Elliott DA, Kirk EP, Yeoh T, Chandar S, McKenzie F, Taylor P, Grossfeld P, Fatkin D, Jones $O$, Hayes $P$, Feneley M, Harvey RP. Cardiac homeobox gene NKX2-5 mutations and congenital heart disease: associations with atrial septal defect and hypoplastic left heart syndrome. J Am Coll Cardiol 2003;41(1 1):2072-6.

10 McElhinney DB, Geiger E, Blinder J, Woodrow BD, Goldmuntz E. NKX2-5 mutations in patients with congenital heart disease. J Am Coll Cardiol 2003;42(9): 1650-5.

11 Erickson RP. Somatic gene mutation and human disease other than cancer. Mutat Res 2003;543(2): 125-36.

12 Youssoufian H, Pyeritz RE. Mechanisms and consequences of somatic mosaicism in humans. Nat Rev Genet 2002;3(10):748-58.

13 Kasahara H, Lee B, Schott JJ, Benson DW, Seidman JG, Seidman CE, Izumo S. Loss of function and inhibitory effects of human CSX/NKX2-5 homeoprotein mutations associated with congenital heart disease. J Clin Invest 2000;106(2):299-308.

14 Freeman SB, Taft LF, Dooley KJ, Allran K, Sherman SL, Hassold TJ, Khoury MJ, Saker DM. Population-based study of congenital heart defects in Down syndrome. Am J Med Genet 1998;80(3):213-7.

15 Loeb LA, Loeb KR, Anderson JP. Multiple mutations and cancer. Proc Natl Acad Sci U S A 2003;100:776-81

16 Meng L, Lin L, Zhang H, Nassiri M, Morales AR, Nadji M. Multiple mutations of the p53 gene in human mammary carcinoma. Mutat Res 1999:435:263-9.

17 Liu W, Cheng J, Iwasaki A, Imanishi H, Hada T. Novel mutation and multiple mutations found in the human butyrylcholinesterase gene. Clin Chim Acta 2002;326:193-9.

18 Westphal V, Schottstadt C, Marquardt T, Freeze HH. Analysis of multiple mutations in the hALG6 gene in a patient with congenital disorder of glycosylation Ic. Mol Genet Metab 2000;70:219-23.

19 Raven KA, Bitts SM, Li L, Golabi M, Cotter PD. Tandem duplication mosaicism: characterization of a mosaic dup(5q) and review. Clin Genet $2001 ; 60(5): 366-70$.

20 Srivastava D, Olson EN. A genetic blueprint for cardiac development. Nature 2000;407(6801):221-6. 